\title{
Modeling of Time of Arrival Method for Lightning Locating Systems
}

\author{
Saeed Vahabi-Mashak, Zulkurnain Abdul-Malek, Kamyar Mehranzamir, \\ Hadi Nabipour-Afrouzi, Behnam Salimi, and Chin-Leong Wooi
}

Institute of High Voltage and High Current (IVAT) and Faculty of Electrical Engineering, Universiti Teknologi Malaysia (UTM), 81310 Johor Bahru, Malaysia

Correspondence should be addressed to Zulkurnain Abdul-Malek; zulkurnain@utm.my

Received 1 August 2014; Revised 5 January 2015; Accepted 5 January 2015

Academic Editor: Zhihua Zhang

Copyright (C) 2015 Saeed Vahabi-Mashak et al. This is an open access article distributed under the Creative Commons Attribution License, which permits unrestricted use, distribution, and reproduction in any medium, provided the original work is properly cited.

\begin{abstract}
Various methods are used to locate cloud-to-ground lightning flashes. Even though a higher cost is incurred, a network of sensor stations is preferable to a single station due to the improved accuracy. For a single station measuring system, the accuracy of its analyses is mostly based on the chosen mathematical equations which can be solved in either linear or nonlinear mode. The sensitivity of the measuring equipment used is also particularly significant. This paper concentrates on the modelling of time of arrival (TOA) technique for locating a lightning flash by utilizing three broadband antennas. Consequently, by employing the developed model, the influences of geometric parameters on the accuracy of the model are evaluated. Therefore, a Matlab based simulation of the measuring system is developed. In the developed codes, randomly located lightning flash with its corresponding electromagnetic radiation was modelled. Results show that parameters such as lightning path shape, distance of the leader, and leader location can directly affect the accuracy of the TOA technique for extracting the azimuth and elevation.
\end{abstract}

\section{Introduction}

Many researches have been done on the modeling of lightning phenomena and its effects. The aims of these researchers are to evaluate, estimate, and analyze various lightning properties and their effects for different applications. Simulation models were developed to cater for all aspects of lightning such as lightning leader and its pattern [1-3], lightning electromagnetic field propagation $[1,4,5]$, lightning induced voltage and current patterns on man-made systems [6-9], and lightning detection and localization using various sensing techniques [1]. However, most of the published works on lightning related simulation models have their own shortcomings. To the best of the authors' knowledge, the developed models are either too limited for specific applications or suitable for addressing only one or two aspects of the lightning. There exists no simulation that includes all aspects of lightning mentioned above.

Some researches were carried out to simulate the general definition of lightning phenomena such as the work done by Clifford et al. [2]. Clifford et al. describe how to simulate a lightning stroke using laboratory equipment. However, because of the limitation of an actual small scale lightning simulation, a numerical simulation can be adopted instead. The numerical models include the work done by Pulgarín et al. [1] and Wan et al. [3] where a procedure to reconstruct the actual lightning leader path as accurate as possible is described. In addition, the simulation work done by Pulgarín et al. also includes an approach to evaluate the effects of lightning induced electric field on the time of arrival technique. This is carried out by reconstructing the tortuosity of the lightning leader path but without considering other lightning parameters such as the induced voltage or current. On the other hand, Wan et al. evaluate the distribution of electric field at the ground level immediately below the stepped leader using a more accurate lightning leader path model.

Simulations considering only specific aspects of lightning were carried out by many researchers. Simulations of lightning induced current on man-made systems, both in time 
and in frequency domains, were carried out by Aniserowicz and Maksimowicz [7], Liu et al. [6], and Gamerota et al. [10]. Similarly, the work done by Gamerota et al. only proposed a simulation procedure to obtain the parameters of the resulting lightning current waveforms without considering the geometry of the lightning leader path or electric field. On the other hand, the lightning electromagnetic field is the focus of modelling done by Delfino et al. [4], Shao and Jacobson [11], and Pulgarín et al. [1]. Simulations of lightning effects on man-made systems were also carried out by Kato et al. [12] and Baba and Rakov [5]. Several specific simulation approaches were developed to evaluate the effect of induced current on fast moving object like aircrafts $[8,13]$, or to estimate inrush currents in power distribution transformers [9]. Some other researchers evaluate the lightning effects on power transmission lines [14] by considering at least two lightning aspects. Phillips and Anderson [15] and Jiang et al. [16] evaluate the overhead transmission line shielding by simulating electric field and lightning leader path properties.

One important modeling aspect is on the lightning detection and localization using various sensing techniques. Several attempts $[1,17-20]$ had been previously carried out to numerically model this aspect of lightning. There are many available methods to detect and localize lightning. One such method is the time of arrival technique.

Time of arrival (TOA) technique, or time of flight, as it is sometimes referred to, is a technique used to determine the location of a lightning radiation source based on the corresponding arrival times at the measuring sensors. Variations of this technique include the time difference of arrival (TDOA) [21], time of group arrival (TOGA) [22], and other methods [23-26]. Each method is suitable to be implemented for a certain application under a specific condition. One possible way to increase the accuracy of the location is to combine two or more locating methods as one measurement system such as the Angle of Arrival (AOA) technique [27] with TDOA, or the AOA with TOA. However, there are difficulties involved in such combined methods system. For each method, the number of sensor stations is critical since it directly affects the accuracy of the generated results [21]. The accuracy can be further increased by using multistation sensors. Generally, several single stations are located around an area of interest.

The TOA technique is usually based on the measurements of difference of arrival times. An example of this is shown in Figure 1(a). Three sensors or antennas are used. The originating source of the signal to be detected is enclosed within the sensors.

This technique has various applications including condition monitoring in substations (e.g., partial discharge location within an oil insulated transformer) and the lightning locating system. The number of sensors could be more than three and this actually depends on the source size and strength, as well as on the desired accuracy of detection. The required locations of the sensors are also dependent on the source location. The accuracy of detection is highly dependent on whether the source is in the center of the coverage area or at its perimeter.

The TOA technique can also be realized using sensors which are located close to each other to form short baselines

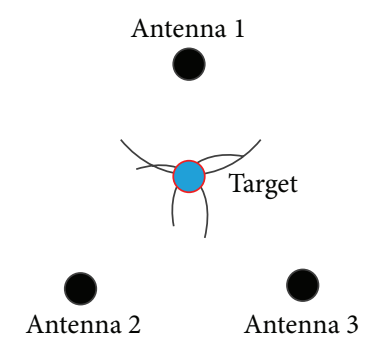

(a)

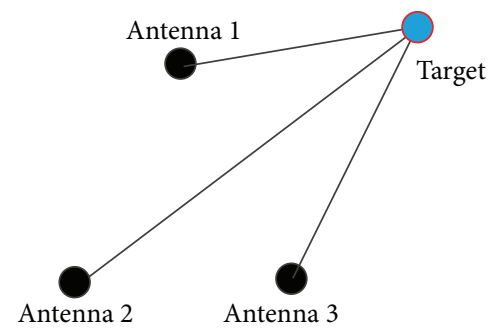

(b)

FIgUre 1: Two types of TOA technique. (a) Object is enclosed within the antennas. (b) Arrangement of three antennas in the short baseline configuration.

with a maximum length of less than 100 meters for a configuration of three antennas, as shown in Figure 1(b). The three-antenna configuration will give the azimuth and elevation angles of the signal source (based on trigonometry principles). A fourth antenna, located at a certain distance away, depending on the measuring limitation, can be added to provide the information needed to determine the exact coordinate $(X, Y, Z)$ of the signal source. This TOA technique is usually used for the purpose of lightning location in a small region (10 to $15 \mathrm{~km}$ radius). Tantisattayakul et al. presented a three-dimensional lightning discharge mapping system using time difference of arrival for VHF lightning pulses. They implemented the 2-D and 3-D lightning discharge models in space [28]. Cao et al. have developed their short baseline technique for investigating lightning discharges. Their system worked well for close lightning discharges. They analyzed the error estimation and the factors that influence on error values in their research [29]. Mardiana and Kawasaki studied the fast moving electromagnetic (EM) radiation during lightning discharges. As one of the results, a cloud-to-ground lightning discharge has been reconstructed in the two-spatial dimension and in time sequence [30]. Salimi et al. implemented the short base line system for lightning locating system using time of arrival method. Their system could map the lightning discharges in 2-D [19, 20]. Mashak et al. simulated the mathematical models for measurement systems based on TOA method to determine the azimuth and elevation angles $[17,18]$.

According to Klebesadel et al., some factors that influence the accuracy of the TOA technique are the mathematical models of measurement system adopted, the length of recorded lightning signal, and the origin of the lightning 
signal (radiation source), waveform reflection due to surrounding structures, and the noise level [31]. Ideally, an accurate TOA based system is implemented in such a way that the effects of all these factors are minimized. The accuracy is very much dependent on the mathematical model used to determine the radiation source. The chosen mathematical model, in turn, may suffer certain errors due to certain simplifying assumptions made during its formulation. A new computer-based ELF/VLF system for locating lightning discharges has been developed by Rafalsky et al. Both the arrival azimuths of atmospherics and the distances to their sources are estimated [32]. Wagenaars et al. evaluated different time of arrival algorithms in order to determine which method yields most accurate location under different circumstances. From the results, both the energy criterion and the phase methods showed the best performance [33].

One common assumption in this TOA technique is that the distance between the radiation source and the sensor station is very much larger than the distance between the sensors within the station. This is similar to the assumption made for the satellite-ground sensor station. Previous studies calculate the azimuth and elevation angles without even considering the possible errors involved due to previously mentioned factors as well as discrepancies in the analysis. This is critical since those angles were directly used in the determination of the lightning coordinates. This paper aims to evaluate the errors involved in the calculation of the azimuth and elevation angles when using a short baseline antenna configuration. It involves the modelling of downward lightning leaders, the corresponding VHF signal propagation, and the TOA technique for locating a lightning downward leader by utilizing three broadband antennas. The influence of geometric parameters on the accuracy of the developed model is evaluated. The simulated model shows that parameters such as lightning path shape, distance of the leader, and leader location can directly affect the accuracy of the azimuth and elevation angles.

\section{Simulation of Lightning Detection Using Short Baseline Configuration}

The simulation of a real lighting signal, taking into account of all physical characteristics, is too complicated to be carried out and it is beyond the scope of this paper. The difficulty of simulation procedure is because of both geometric shape and electromagnetic specifications of a lightning flash. The following procedure shows 12 important steps in the simulation process which can be divided into three main sections, namely, the calculation and plotting of the coordinates along the leader's path (steps (1)-(7)), the determination and plotting of the induced voltage waveforms (steps (8)(9)), and finally the calculation of the azimuth and elevation angles (steps (10)-(12)). Firstly, we need to determine the lightning leader's path. This is described in the following steps (1)-(7).

(1) Obtain two random coordinates in three dimensional spaces, marked as starting and ending points of a lightning leader (Figure 2):

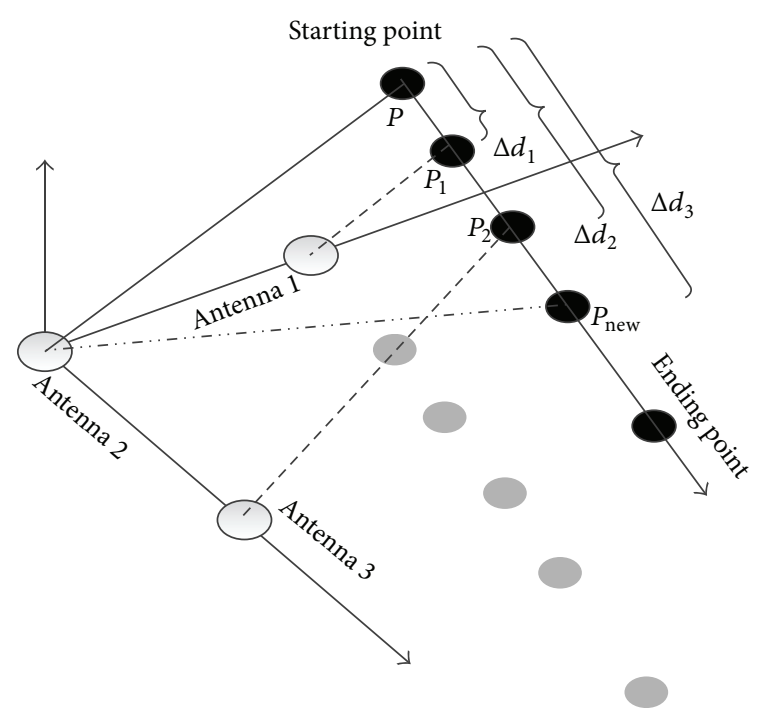

FIGURE 2: Schematic description of the geometry of three antennas and the position of radiation source.

\author{
starting point: $\left(X_{S}, Y_{S}, Z_{S}\right)$; \\ ending point: $\left(X_{E}, Y_{E}, Z_{E}\right)$; \\ the coordinates of the receiving antennas are \\ antenna 3: $(14.5,0,0)$, \\ antenna $2:(0,0,0)$, \\ antenna $1:(0,14.5,0)$.
}

(2) Draw a straight-line by using the parametric equation of a line in 3D between the two points [34]:

$$
(X, Y, Z)=\left(X_{S}, Y_{S}, Z_{S}\right)+t \times\left(\left(X_{E}, Y_{E}, Z_{E}\right)-\left(X_{S}, Y_{S}, Z_{S}\right)\right)
$$

where $t$ is a parameter with values between 0 and 1 .

(3) Using the starting point as the reference coordinate:

$$
\text { starting point: }\left(X_{S}, Y_{S}, Z_{S}\right)=\left(X_{P}, Y_{P}, Z_{P}\right) \text {. }
$$

(4) Obtain an unknown point, say $P_{1}$, on the straightline to satisfy the governing equation between antenna 2 and antenna 1 as given by

$$
\begin{aligned}
\left(X_{P 1}, Y_{P 1}, Z_{P 1}\right)= & \left(X_{S}, Y_{S}, Z_{S}\right) \\
& +t \times\left(\left(X_{E}, Y_{E}, Z_{E}\right)-\left(X_{S}, Y_{S}, Z_{S}\right)\right)
\end{aligned}
$$

To solve (2), $t$ needs to be determined (described in (5)), which in turn requires us to solve (3) and (4). A radiation source can be segmented into infinitesimal portions. Assume a radiation source exists at $P$ (Figure 2) and moments later another portion appears at $P_{1}$. It is noted that while antenna 2 is capturing the portion at $P$ when it is first triggered, antenna 1 may capture the signal at $P_{1}$ due to the different times of arrival. Equations (3) and (4) describe the relationships between various distances as in Figure 2. Finally, $t$ can be calculated using (5). 
Let

$$
\begin{gathered}
d_{0}=\sqrt{X_{S}^{2}+Y_{S}^{2}+Z_{S}^{2}}, \\
d_{P 1}=\sqrt{\left(X_{P 1}-14.5\right)^{2}+Y_{P 1}^{2}+Z_{P 1}^{2}}, \\
\Delta d_{1}=\sqrt{\left(X_{P 1}-X_{S}\right)^{2}+\left(Y_{P 1}-Y_{S}\right)^{2}+\left(Z_{P 1}-Z_{S}\right)^{2}} .
\end{gathered}
$$

Equation (4) shows how far an electromagnetic waveform can travel, first along the main path $\Delta d$ (from $P$ to $P_{1}$ ), second from point $P_{1}$ to a destination such as antenna 1 . The summation of the two passed path should become the same as the distance where electromagnetic waveform travels from point $P$ to the antenna 2 .

Then,

$$
\begin{aligned}
& d_{0}=d_{P 1}+\Delta d_{1}, \\
& t=\frac{\left(-B+S^{2}\right)}{(2 \times D \times S+C)} .
\end{aligned}
$$

The parameters can be obtained as below.

Let

$$
\begin{gathered}
\alpha=X_{E}-X_{S}, \quad \beta=Y_{E}-Y_{S}, \\
\gamma=Z_{E}-Z_{S}, \quad K=X_{S}-14.5, \\
F=X_{S}-0, \quad G=X_{S}-0 .
\end{gathered}
$$

Then,

$$
\begin{gathered}
A=\alpha^{2}+\beta^{2}+\gamma^{2}, \quad B=K^{2}+F^{2}+G^{2}, \\
C=2 \times(\alpha \times K+\beta \times F+\gamma \times G), \\
S=d_{0}, \quad D=\sqrt{A} .
\end{gathered}
$$

(5) Obtain another unknown point, say $P_{2}$, on the straight-line to satisfy the governing equation between antenna 2 and antenna 3 as given by (8) and parameter $t$ can be calculated using (12) as follows:

$$
\begin{gathered}
\left(X_{P 2}, Y_{P 2}, Z_{P 2}\right)=\left(X_{S}, Y_{S}, Z_{S}\right) \\
+t \times\left(\left(X_{E}, Y_{E}, Z_{E}\right)-\left(X_{S}, Y_{S}, Z_{S}\right)\right), \\
d_{P 2}=\sqrt{X_{P 1}^{2}+\left(Y_{P 1}-14.5\right)^{2}+Z_{P 1}^{2}}, \\
\Delta d_{2}=\sqrt{\left(X_{P 2}-X_{S}\right)^{2}+\left(Y_{P 2}-Y_{S}\right)^{2}+\left(Z_{P 2}-Z_{S}\right)^{2},} \\
d_{0}=d_{P 2}+\Delta d_{2}, \\
\alpha=X_{E}-X_{S}, \quad \beta=Y_{E}-Y_{S}, \\
\gamma=Z_{E}-Z_{S}, \quad K=X_{S}-0, \\
F=X_{S}-14.5, \quad G=X_{S}-0, \\
t=\frac{\left(-B+S^{2}\right)}{(2 \times D \times S+C)} .
\end{gathered}
$$

(6) Determine a new reference point $\left(P_{\text {new }}\right)$ on the straight-line using (13) and parameter $t$ can be calculated using (18).

For a $100 \mathrm{MS} \mathrm{s}^{-1}$ sampling rate, a gap between samples is equivalent to a short electromagnetic wave propagation distance proportional to speed of light. This is finally translated into a $3 \mathrm{~m}$ distance as written below:

$$
\begin{aligned}
& \left(X_{P_{\text {new }}}, Y_{P_{\text {new }}}, Z_{P_{\text {new }}}\right)=\left(X_{S}, Y_{S}, Z_{S}\right) \\
& +t \times\left(\left(X_{E}, Y_{E}, Z_{E}\right)-\left(X_{S}, Y_{S}, Z_{S}\right)\right), \\
& d_{0}=\sqrt{X_{S}^{2}+Y_{S}^{2}+Z_{S}^{2}} \\
& d_{0_{\text {new }}}=\sqrt{X_{P_{\text {new }}}^{2}+Y_{P_{\text {new }}}^{2}+Z_{P_{\text {new }}}^{2}} \text {, } \\
& \Delta d_{3}=\sqrt{\left(X_{P_{\text {new }}}-X_{S}\right)^{2}+\left(Y_{P_{\text {new }}}-Y_{S}\right)^{2}+\left(Z_{P_{\text {new }}}-Z_{S}\right)^{2}} \text {, } \\
& d_{0_{\text {new }}}=d_{0}+3 \mathrm{~m} .
\end{aligned}
$$

The parameter can be obtained as below:

$$
\begin{gathered}
\alpha=X_{E}-X_{S}, \quad \beta=Y_{E}-Y_{S}, \\
\gamma=Z_{E}-Z_{S}, \quad K=X_{S}-0, \\
F=X_{S}-0, \quad G=X_{S}-0, \\
A=\alpha^{2}+\beta^{2}+\gamma^{2}, \quad B=K^{2}+F^{2}+G^{2}, \\
C=2 \times(\alpha \times K+\beta \times F+\gamma \times G), \\
P=\sqrt{B}, \quad D=\sqrt{A}, \\
S=P+3.3, \quad t=\frac{\left(-B+S^{2}\right)}{(2 \times D \times S+C)} .
\end{gathered}
$$

(7) Repeat steps (3)-(6) to obtain the desired number of coordinates. The desired number of coordinates is dependent on $t$, which means calculations are stopped when $t$ is larger than one.

In each loop (steps (3)-(6)), three new coordinates $\left(P_{1}\right.$, $P_{2}$, and $\left.P_{\text {new }}\right)$ are calculated. This loop for calculating new coordinates should be continued until any $t$ (obtained from (5), (12), and (18)) becomes negative or larger than one. In addition, after each loop, another check should be carried out to ensure the calculation remains valid (calculated coordinates are within the leader length with the starting coordinate $\left(X_{S}, Y_{S}, Z_{S}\right)$ and ending coordinate $\left.\left(X_{E}, Y_{E}, Z_{E}\right)\right)$ as given in (1). Therefore, to test the condition ( $t$ should remain between zero and one), the rearranged equation number one presented in (19) should be used. In (19), $(X, Y, Z)$ should be substituted by the coordinates of each $P_{1}, P_{2}$, and $P_{\text {new }}$. $\left(X_{S}\right.$, $\left.Y_{S}, Z_{S}\right)$ and $\left(X_{E}, Y_{E}, Z_{E}\right)$ values are as in (1):

$$
t=\frac{\left((X, Y, Z)-\left(X_{S}, Y_{S}, Z_{S}\right)\right)}{\left(\left(X_{S}, Y_{S}, Z_{S}\right)-\left(X_{E}, Y_{E}, Z_{E}\right)\right)} .
$$




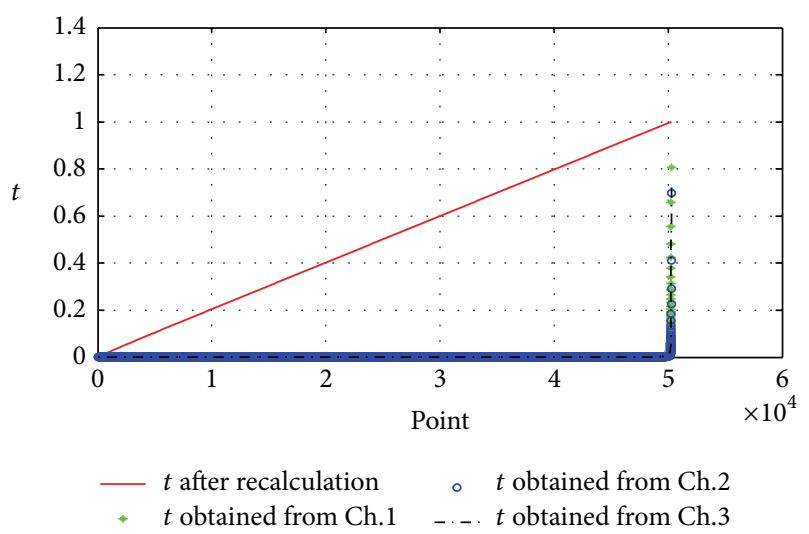

FIGURE 3: Variation of $t$ values with iteration number for the purpose of simulation check. The simulation is stopped when any $t$ exceeds one.

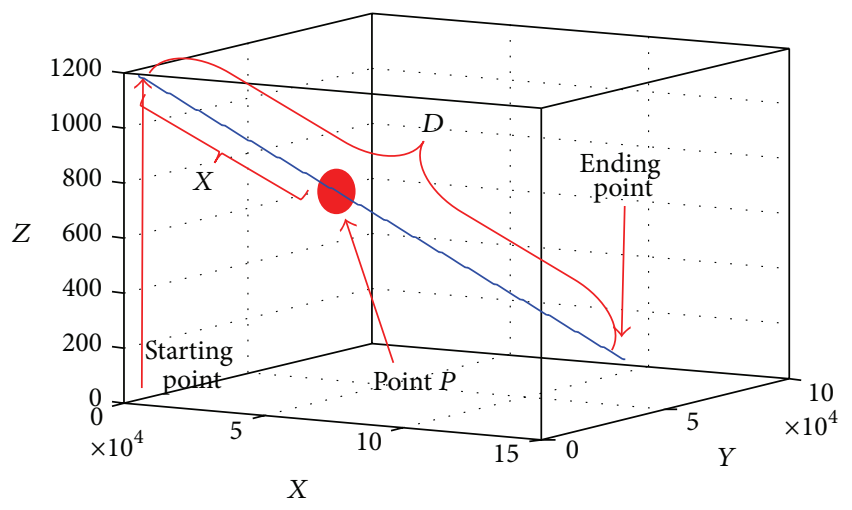

FIgURE 4: A linear path of simulated lightning leader.

The variation of parameter $t$ from all equations with the iteration number can be seen in Figure 3. The parameter $t$ calculated for each antenna is mostly small (but positive) except at the last tens of iterations. On the other hand, the $t$ value from (19) calculated at the end of each loop linearly increases from 0 to 1 .

Once the ending coordinate $\left(X_{E}, Y_{E}, Z_{E}\right)$ (as in (1)) is reached, sufficient coordinates which represent the path travelled by the leader are obtained. This is shown in Figure 4.

The next thing to do is to simulate the captured signals by all three antennas as a time varying signal or waveform. This is described in steps (8)-(9).

(8) Choose a radiation signal waveform to represent a lightning leader.

In this work, a $14.35 \mathrm{kHz}$ sinusoidal waveform was chosen. For a four-cycle signal, an equivalent of $8.362 \mathrm{~km}$ distance $D$ travelled by the leader was obtained assuming the velocity is one tenth of the speed of light, and corresponding 50273 successive points or coordinates were generated for each channel. We can assume our ending point defined earlier as the coordinate where the leader almost reaches the ground and therefore can be assumed equal to the return stroke coordinate.

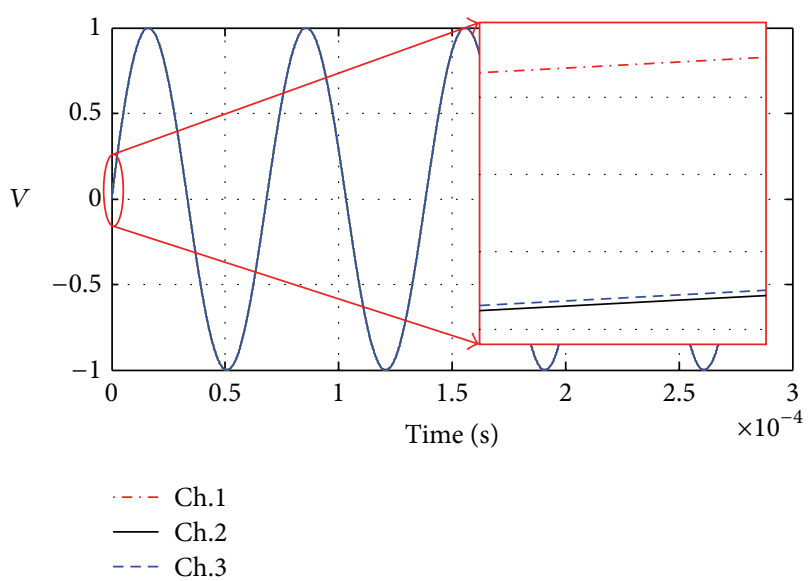

FIGURE 5: The captured signals for all antennas overlapping each other. The captured signals after time scale magnification where the delays between antennas can be clearly inferred.

(9) Determine the magnitude of the captured signal by first calculating the distance between each calculated coordinate and the starting point (this distance in labelled as $x)$.

The captured voltage magnitude at each antenna can then be calculated. In this work, this magnitude is given as $\sin (8 \pi x / D)$ where $D$ is the length of the signal path in $\mathrm{m}$, and $x$ is the distance of such a point $P$ to starting point as shown in Figure 4.

By considering all coordinates and hence the values of $x$, a sinusoidal waveform as a function of time is obtained. This is shown in Figure 5.

Steps (10)-(12) describe the procedures for calculating the azimuth and elevation angles.

(10) Segment each captured waveform into a certain number of segments. Each segment contains three different antenna signals.

(11) Determine the delay times in each segment. These are given as

$t_{21}=$ the delay between antenna 2 and antenna 1 for receiving signal;

$t_{23}=$ the delay between antenna 2 and antenna 3 for receiving signal. Details of the calculation are determined later in the paper.

(12) Calculate the azimuth and elevation angles for each segment of the waveforms. To calculate the azimuth $(\alpha)$ and the elevation $(\beta)$ of a lightning leader by using the shortbaseline TOA technique, the following proposed time based equations are to be used [28]:

$$
\begin{gathered}
\alpha=\tan ^{-1}\left(\frac{t_{23}}{t_{21}}\right), \\
\beta=\cos ^{-1}\left(\left(\frac{c}{d}\right) \times \sqrt{\left(t_{23}^{2}+t_{21}^{2}\right)}\right) .
\end{gathered}
$$

The parameter $d$ is the length of the short baseline which is 14.5 meters. 


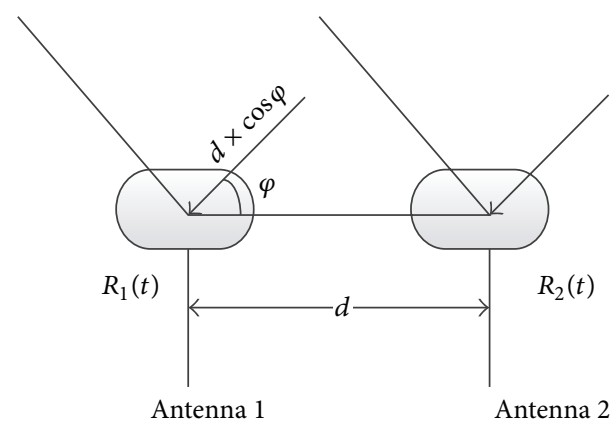

FIGURE 6: The position of radiation source and two flat parallel broadband antennas. Also, the assumption of having parallel radiated signals.

This section has described the simulation of lightning detection using short baseline configuration. The next few sections discuss the evaluation of the accuracy of the azimuth and elevation angles determination using baseline antennas. In particular, the effects of distance of the lightning leader from the baseline antennas, of the shape of the leader's path, and of the location of the leader with respect to the baseline antennas, on the accuracy of the delay time calculation, are described.

\section{Effects of Distance}

In this section, the effects of the distance of the lightning leader from the baseline antennas on the accuracy of the azimuth and elevation angles are described. To determine the azimuth $(\alpha)$ and the elevation $(\beta)$ of a lightning event by using TOA method, the above time based equations (20) and (21) are used.

The origin of the radiation source is assumed to be quite far from the baseline antennas compared to the distance between two antennas, or $d$, as shown in Figure 6. This is the fundamental assumption made in deriving the equations for $\alpha$ and $\beta$ in (20) and (21).

By assuming a very large distance of lightning source, two parallel lines of radiation arriving on two flat antennas can be drawn (Figure 6). However, the derivation of $\alpha$ and $\beta$ ((20) and (21)) based on this parallel line configuration is erroneous for a coverage distance of typical baseline antennas which is about $50 \mathrm{~km}$. A distance of more than $100 \mathrm{~km}$ is required for the equations to be correctly used without significant errors.

The delay times $\left(t_{21}\right.$ and $t_{23}$ ) (for each loop (steps (3)-(6) in Section 2)), which were calculated using (22), in terms of $d_{1}, d_{2}$, and $d_{3}$, are defined as follows:

$d_{1}$ : the distance between $P_{\text {new }}$ and antenna 1 , $d_{2}$ : the distance between $P_{\text {new }}$ and antenna 2, $d_{3}$ : the distance between $P_{\text {new }}$ and antenna 3,

$$
\begin{aligned}
& t_{21}=\frac{\left(d_{2}-d_{1}\right)}{c}, \\
& t_{23}=\frac{\left(d_{2}-d_{3}\right)}{c},
\end{aligned}
$$

$c$ is the speed of light.

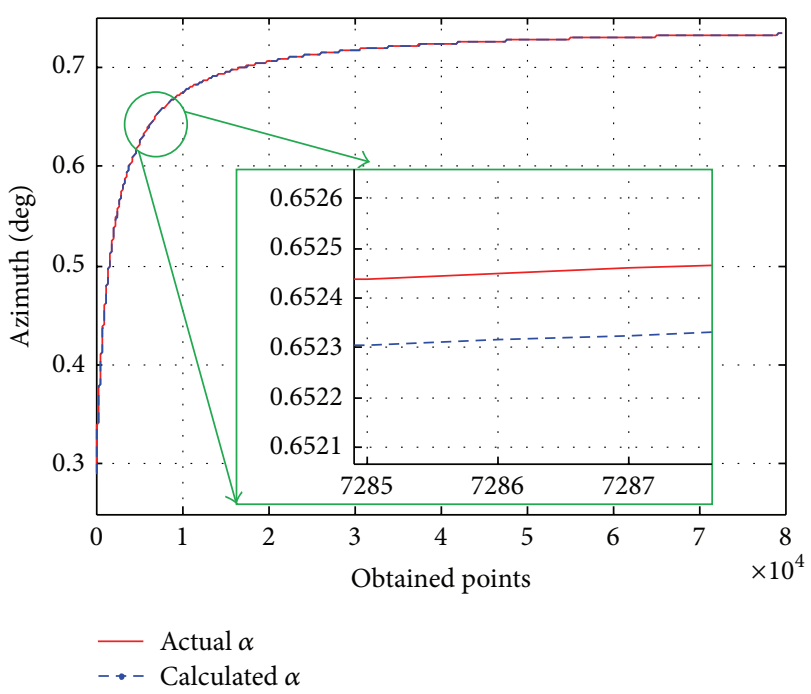

FIGURE 7: The variation of both the actual azimuth, $\alpha$, which is obtained geometrically, and the calculated $\alpha$, which is determined using time delays.

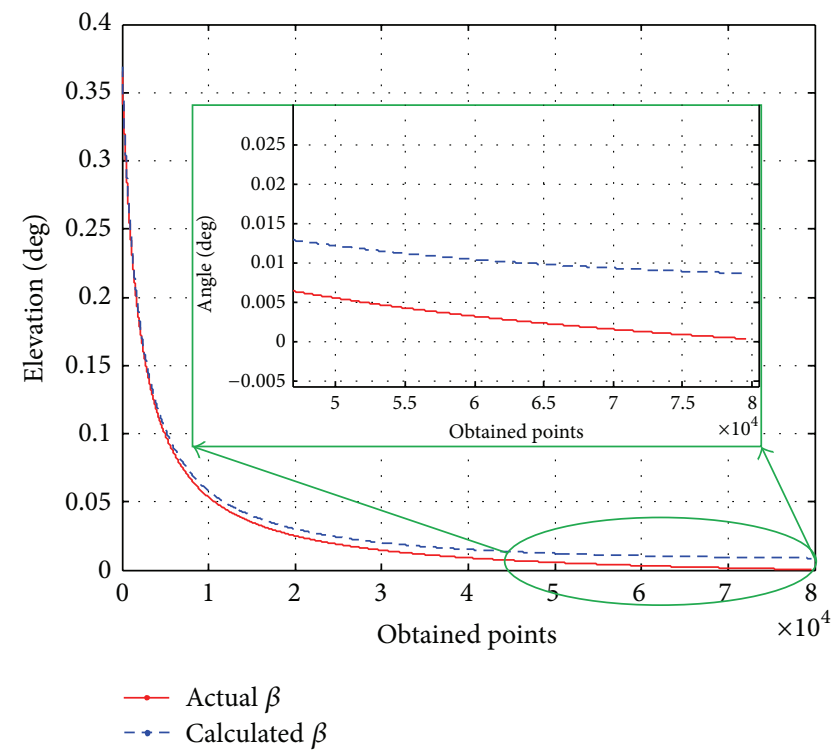

FIGURE 8: The variation of the actual elevation, $\beta$, which is geometrically determined, and the calculated $\beta$ (calculated using time delays).

$\alpha$ and $\beta$ were then calculated and plotted (labelled as calculated) as shown in Figures 7 and 8.

To illustrate the error, $\alpha$ and $\beta$ values were again determined using the known geometric equations as follows:

$$
\begin{gathered}
\alpha=\tan ^{-1}\left(\frac{Y}{X}\right), \\
\beta=\tan ^{-1}\left(\frac{Z}{\sqrt{\left(X^{2}+Y^{2}\right)}}\right) .
\end{gathered}
$$




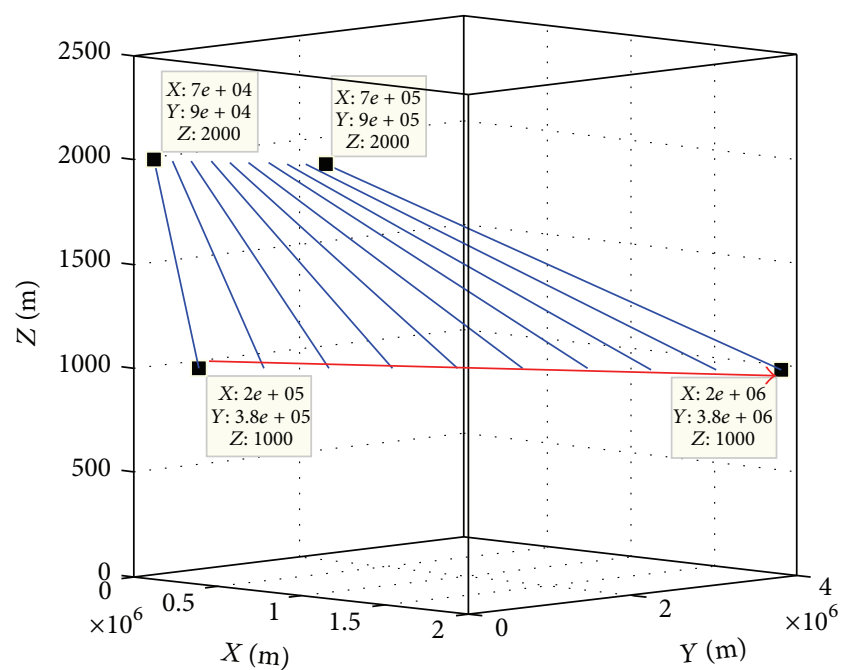

FIGURE 9: Simulated lightning leader at various distances from the sensor station to determine its effect on the model computation errors.

These values of $\alpha$ and $\beta$ are the actual values. They are also plotted in Figures 7 and 8 with the "actual" label. As shown in Figure 7, the calculated $\alpha$ using the time delay equation compares well with the actual values using the geometric equation. The error is in the order of hundreds of microdegrees. On the other hand, there is a relatively large error in the case of $\beta$ as shown in Figure 8. The error is in the order of hundreds of millidegrees. This is translated into hundreds of meter leader distance, which is significant. To evaluate the effect of changing the distance of a radiation source from baseline antennas on the error in $\alpha$ and $\beta$, simulations were carried out with varying leader path location in 1000 steps. The following first and final locations (in meters) were used:

\section{first location:}

starting point: $(700,900,2000)$,

ending point: $(2000,3800,1000)$;

final location:

starting point: (7E5, 9E5, 2000),

ending point: $(2 \mathrm{E} 6,3.8 \mathrm{E} 6,1000)$.

A total of 1000 leader paths were generated, and samples of these are shown in Figure 9.

The average errors for $\alpha$ and $\beta$ as the location of the leader is varied are shown in Figures 10 and 11. The average error for $\alpha$ changes from $0.07^{\circ}$ at $700 \mathrm{~m}$ leader location to almost $0^{\circ}$ at $4000 \mathrm{~km}$ leader location. But, the average error for $\beta$ changes from $0.26^{\circ}$ at $700 \mathrm{~m}$ leader location to almost $0.09^{\circ}$ at $4000 \mathrm{~km}$ leader location.

The simulation results shown in Figures 10 and 11 give the average error for an azimuth variation from 52 to 62 degrees. To evaluate the model for all azimuth angles and all possible elevation angles, instead of using a line, the simulation was repeated for a circular leader path (to provide a $360^{\circ}$

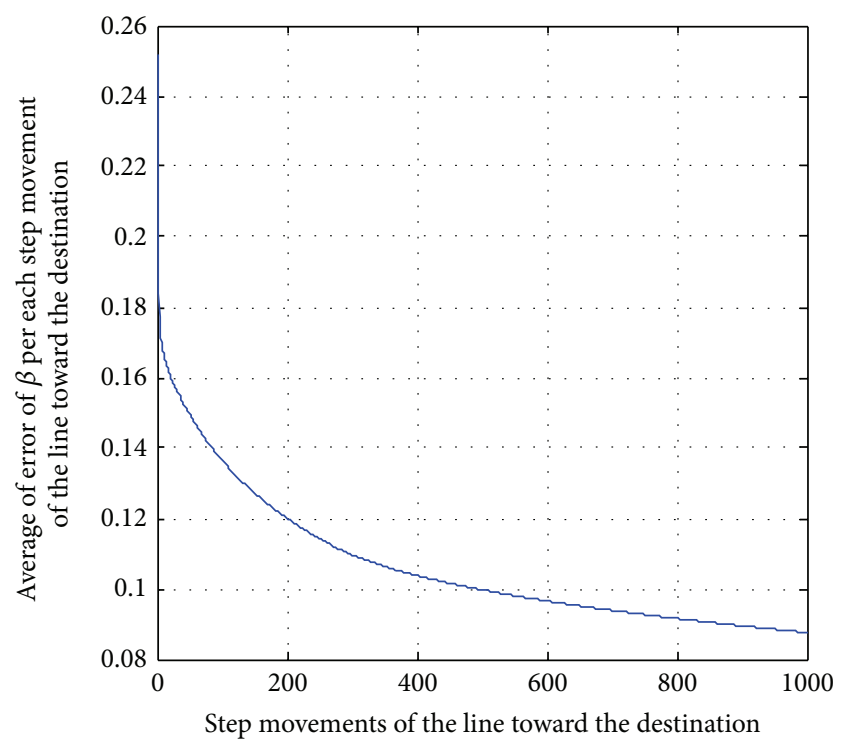

FIgURE 10: The variation of (average) error in degrees for $\beta$ with distance (shown in step movements away from the sensor up to $4000 \mathrm{~km})$.

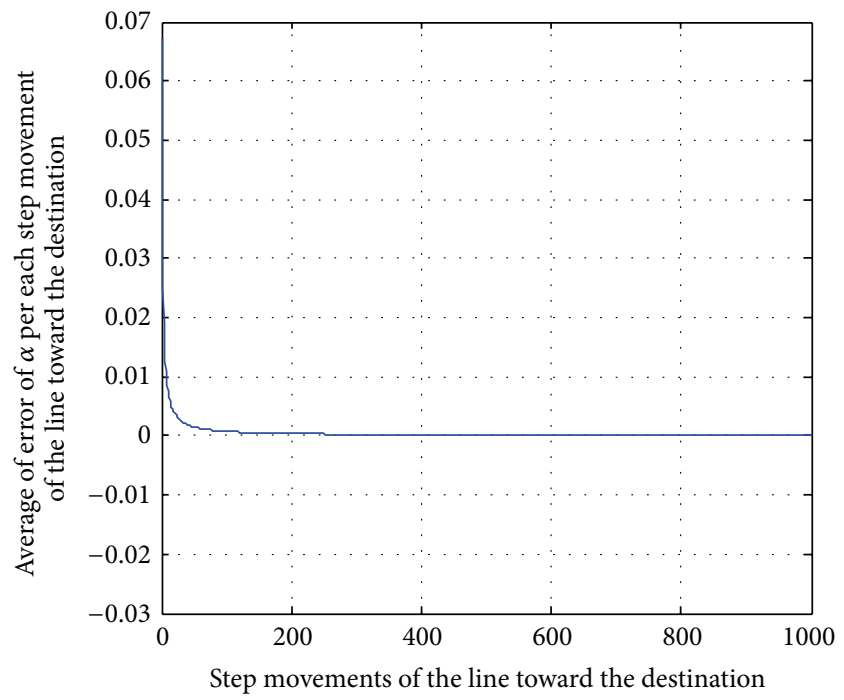

FIGURE 11: The variation of (average) error in degrees for $\alpha$ with distance (shown in step movements away from the sensor up to $4000 \mathrm{~km}$ ).

azimuthal variation with a resolution of $1^{\circ}$ ) for changing radius of $1 \mathrm{~km}$ to $4000 \mathrm{~km}$ (with 34 steps), and for variable elevation from $10^{\circ}$ to $87^{\circ}$ (with 8 steps). Figure 12 shows the maximum error of $\alpha$ for a 360-degree rotation and the distance from the leader to the antennas varied from $1 \mathrm{~km}$ to $4000 \mathrm{~km}$. Each line presented in Figures 12 and 13 represents a unique elevation. Figure 13 shows the maximum error of $\beta$ for all azimuth angles. The evaluation demonstrated in Figures 12 and 13 shows that the maximum error of $\alpha$ happens when the lightning leader has an elevation $\beta=87^{\circ}$, which is high elevation. In contrast, the maximum error of $\beta$ is when $\beta$ $=10^{\circ}$, which is low elevation. As it will be demonstrated in 


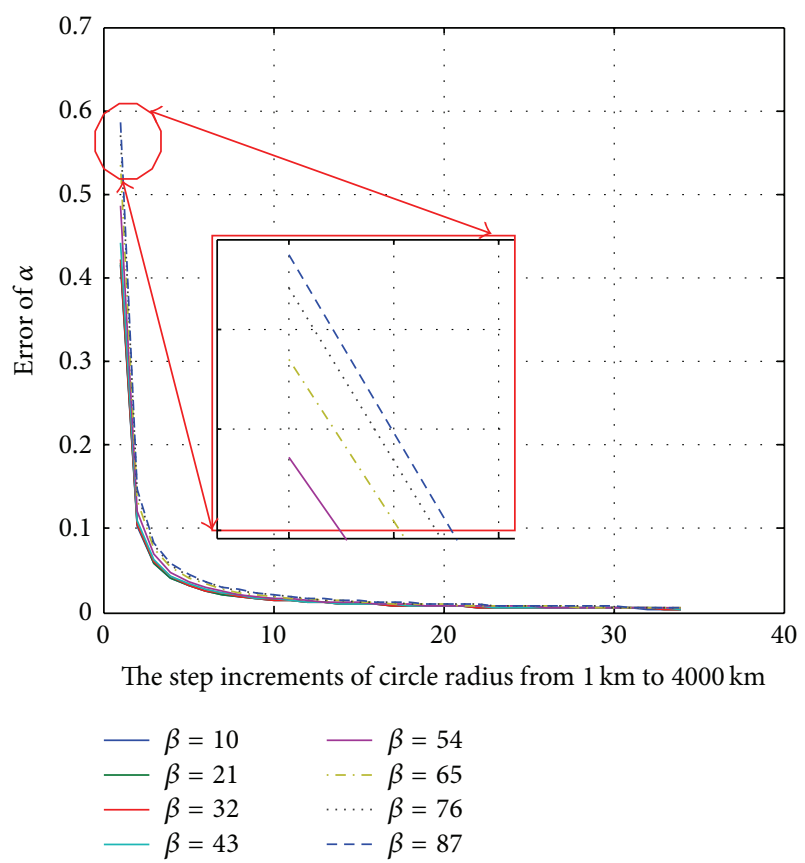

FIGURE 12: The maximum error of $\alpha$ in a complete 360-degree rotation of the azimuth of the lightning source while the distance to antenna sensors is varied from $1 \mathrm{~km}$ to $4000 \mathrm{~km}$ with 34 steps for 8 different elevation levels.

Section 5, the most severe cases of error in the calculation of $\alpha$ and $\beta$ happen in very low or very high elevation, at which conditions the TOA model fails.

\section{Effects of Lightning Path Shape}

The effects of the shape of the leader's path on the accuracy of the azimuth and elevation angles are described in this section. Lightning phenomenon occurs in the form of stepped leader and branched streamers as the leader propagates from the cloud to the oppositely charged earth [35]. In the previous section, this path was represented as a linear path. Therefore the delay time calculation based on the peak detection of the antenna voltages can be easily and correctly calculated. However, for the case of nonlinear and complex leader's path, the calculation will be more complicated since the delay times $\left(t_{21}\right.$ and $\left.t_{23}\right)$ are very much dependent on the instantaneous locations of the radiation source. Since the shape and geometry of leader channels affect the accuracy of the signal processing methods, this shape effect must be included in the actual calculation of the angles.

Figure 14 shows a lightning leader's path in the form of a helix to simulate a complex leader's path. The helix path was chosen in order to magnify the effect of all possible variations in the leader shape. Figure 15 shows the calculated distance of each point on the helical path shown in Figure 14 with respect to the three broadband antennas. As can be seen in Figure 14, the distance between a point on the path and a given antenna is oscillating as the calculation is done along the leader's path. In other words, the resultant delay times could be either positive or negative. This is further illustrated in Figure 16. If

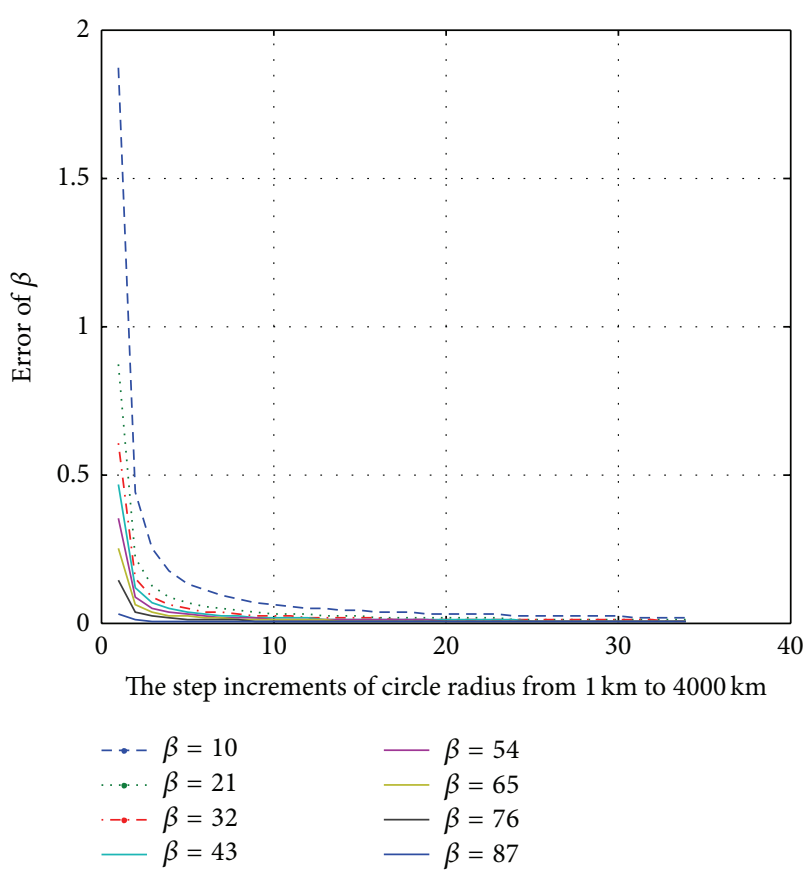

FIGURE 13: The maximum error value of $\beta$ in a complete 360 -degree rotation of the azimuth of lightning source while the distance to antenna sensors is varied from $1 \mathrm{~km}$ to $4000 \mathrm{~km}$ with 34 steps for 8 different elevation levels.

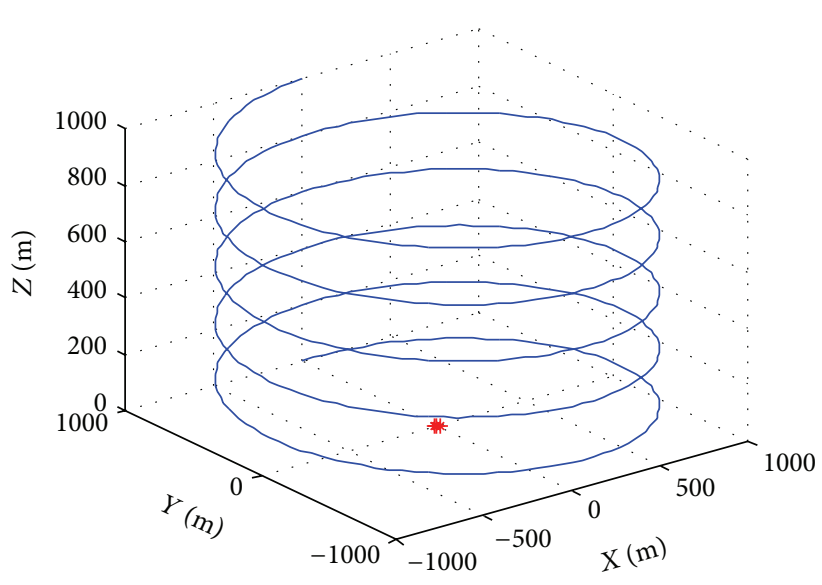

FIGURE 14: A simulated lightning leader's path in the form of a helix.

the distance of a nominal point to the antenna 1 is $D_{1}$ and that to the antenna 3 is $D_{3}$, then, based on the geometry, in some parts $D_{1}>D_{3}$ and in some other parts $D_{3}>D_{1}$. As a result, the time differences, $t_{21}$ and $t_{23}$, dynamically change. Therefore, the calculated distance versus time is also changing dynamically as we expected.

Figure 17 presents semireal shapes of lightning path, including some branches. Each branch independently transmits a signal simultaneously with other branches. Simultaneous transmission of signals from all the leader branches including the main one causes interference in the received or captured signals. The variation is a key factor in the 


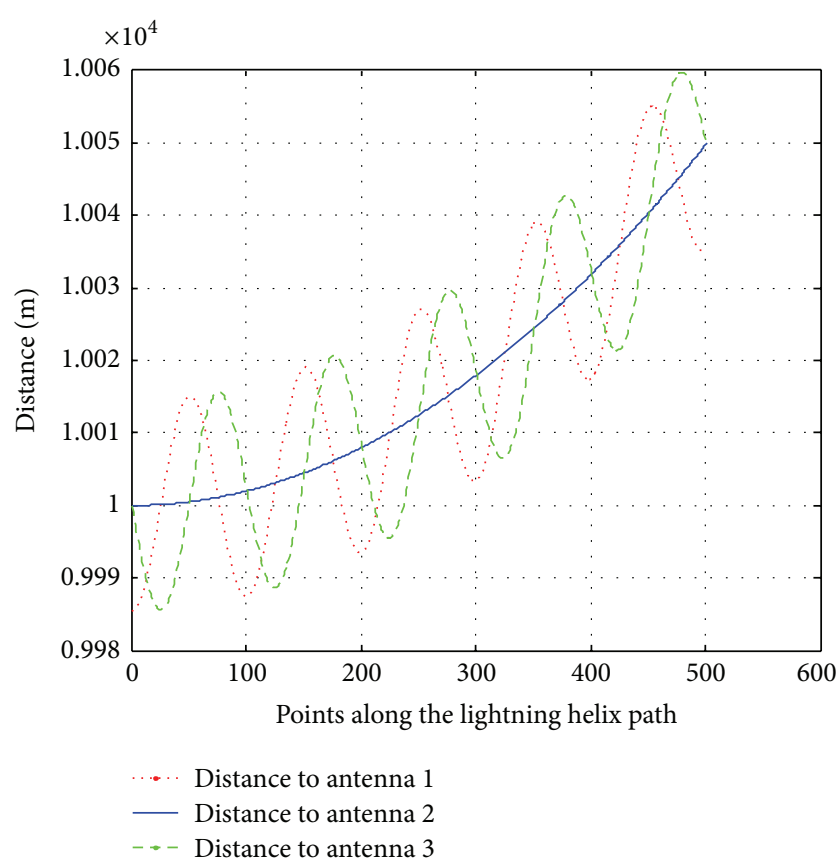

FIGURE 15: Computed distance between points along a helical path of lightning and the antennas at the sensor station.

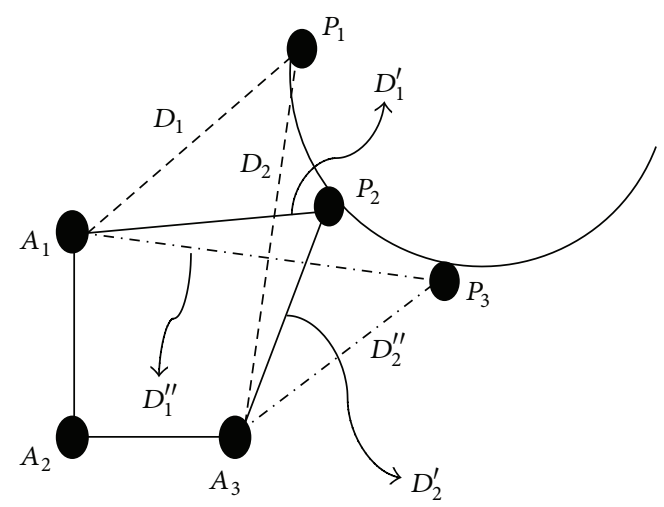

FIGURE 16: A figure to illustrate how the resultant delay times could be either positive or negative. A small portion of the lightning path shown in Figure 14 is redrawn here. Moving from $P_{1}$ to $P_{3}$, first at $P_{1}$, $D_{2}>D_{1}$, then at $P_{2}, D_{2}=D_{1}$, and finally at $P_{3}, D_{2}<D_{1}$.

procedure of finding the delay time in such a method as crosscorrelation which is dependent on the definition of a window of digital data as input data for signal processing.

\section{Effects of Leader Location and Locus of Error}

In this section, the effects of the location of a given leader's path on the accuracy and even the validity of the calculated angles are discussed. The space surrounding the sensor station can be divided into four quadrants. As previously mentioned, there are limitations of the main equations used in the TOA technique, namely, (20) and (21). It is required to

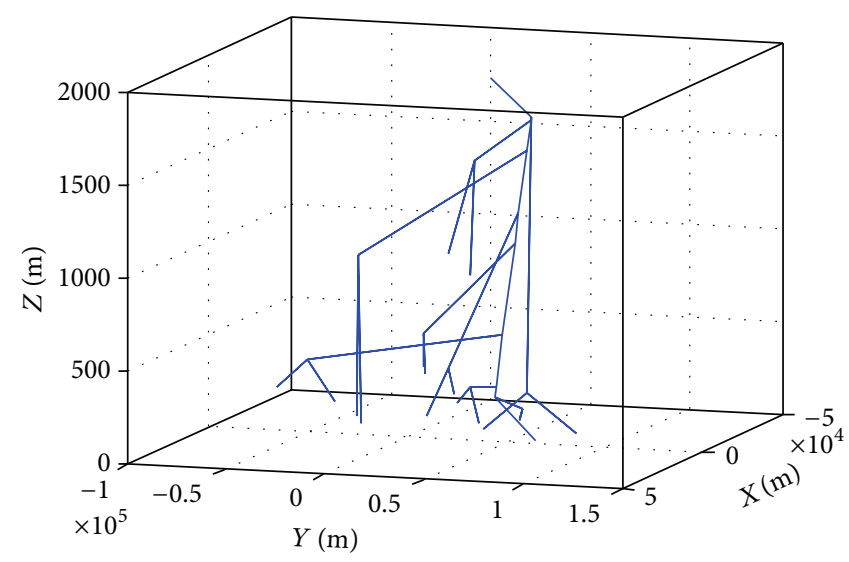

FIGURE 17: Semireal shape of lightning path, including some branches.

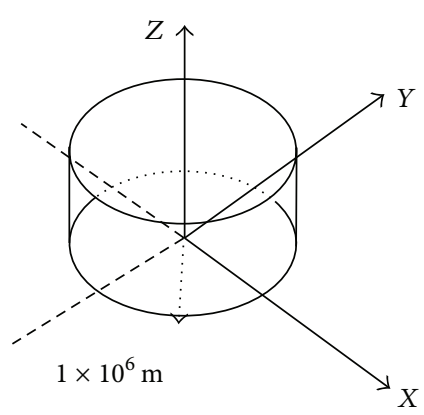

FIGURE 18: An example of many cylindrical loci for evaluating the TOA method.

evaluate the validation of those two equations in the 3D space. To evaluate the validity of the TOA mathematical model, the method was assessed at 360 million points inside of a cylindrical locus expanded from a radius of 100 meters to a radius of $1 \times 10^{6}$ meters as shown in Figure 18. Figure 19 shows the locus in which TOA technique fails because of mathematical limitation of the model. When the argument in (21) is greater than 1 or less than -1 , the TOA technique fails.

As Figure 20 illustrates, most errors occur in the third quadrant (258,649 points), followed by the fourth $(32,573$ points), the second (32,653 points), and the first quadrant (20 points). The errors occur in the simulation as the azimuth changes from $0^{\circ}$ to $360^{\circ}$ and the elevation varies from $0^{\circ}$ to $88^{\circ}$. The maximum $88^{\circ}$ for the elevation was chosen, firstly because of limitation in (20), and secondly because a $90^{\circ}$ angle accrues at the top of the short baselines. The simulation was set up with steps of one degree for both the azimuth and the elevation. However, for two last degrees of the elevation, very fine steps were necessary. The presented data in Figure 20 does not include errors which may accrue from angles $88^{\circ}$ to $90^{\circ}$. Figure 21 shows the distribution of azimuth and elevation angles for which the TOA equation cannot be solved. Based on what is presented in Figure 21, it is necessary to have a complementary measuring equipment to detect the direction of lightning source. Otherwise, correct 


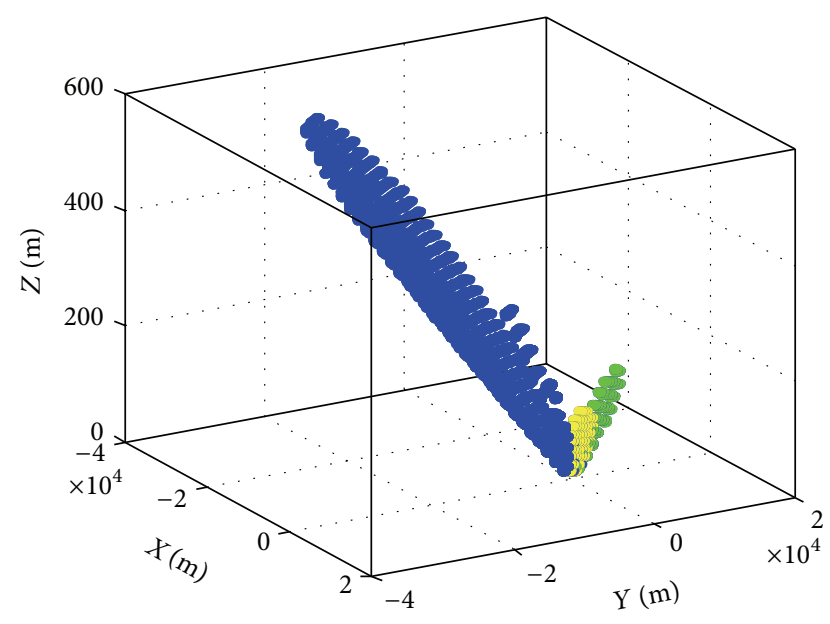

Figure 19: 3D locus of coordinates in which the TOA equation cannot be solved.

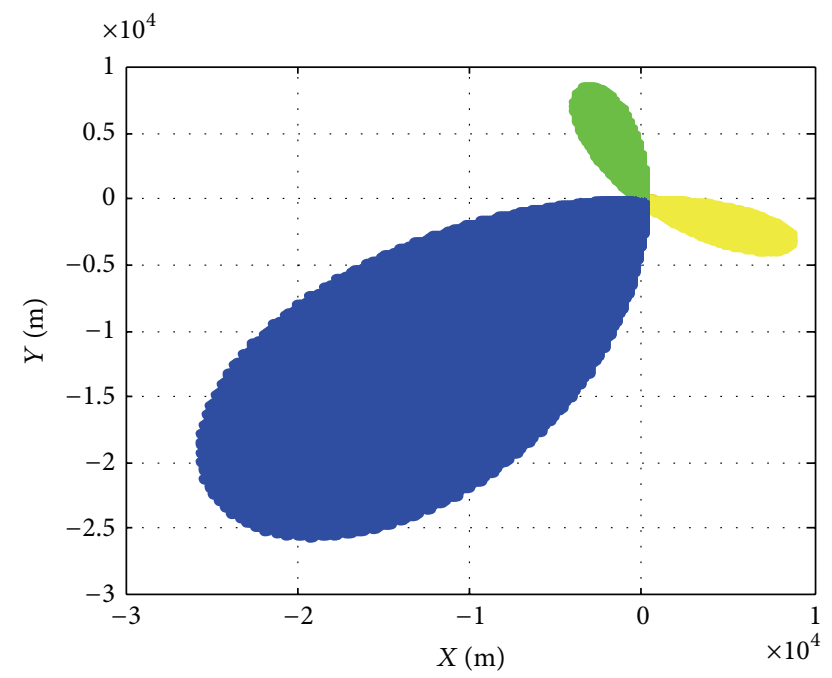

FIgURE 20: The $X-Y$ coordinates in which the TOA equation cannot be solved (within $2 \mathrm{nd}, 3 \mathrm{rd}$, and 4 th quadrants).

and accurate detection of the azimuth and elevation cannot be ascertained. That is to say, the system works properly when the cloud-ground discharges are in the first quadrant.

In this section and the two previous sections, the effects of parameters such as location, path shape, and distance of a leader were evaluated. Those parameters can be grouped as the natural parameters or characteristics of a lightning leader.

\section{Discussion}

The simulation model proposed in this paper addresses the transmission of signals from a lightning leader and the capturing of those signals by a faraway detecting sensor in the form of two short baselines. It also analyses the pattern of arrival and the accuracy of the computed azimuth and elevation angles. To the best of the authors' knowledge, there is no similar previous work that can be used for

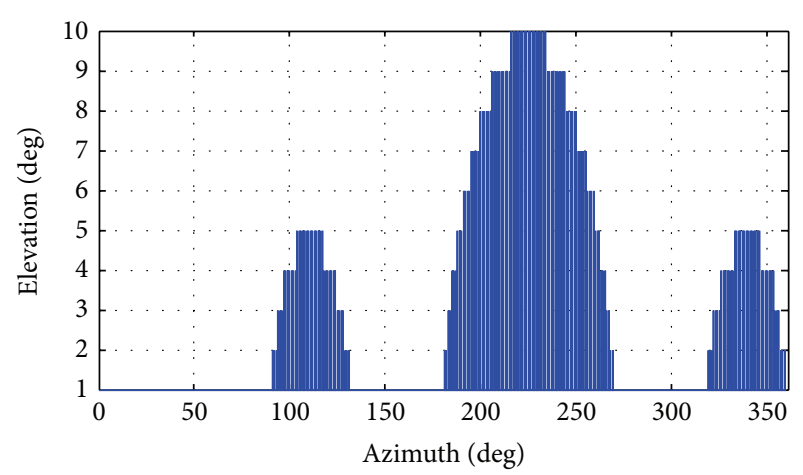

FIGURE 21: The distribution of azimuth and elevation angles for which the TOA equation cannot be solved.

a direct comparison. Nevertheless, this section describes several possible comparisons pertaining to certain aspects of the lightning simulation.

The work by Gamerota et al. [10] describes the effect of lightning signal models used. For example, a double exponential impulse signal is more representative of the actual lightning signal. However, in the current work, a sinusoidal type signal was used because of two reasons: firstly, to increase the occurrence probability of a nonlinear time delay for the purpose of its evaluation; secondly, because only the time delay of signal arrivals is important in the TOA technique. The shape of induced current can be neglected as long as a proper technique is employed for the nonlinear time delay extraction.

As discussed in Section 4, the effect of lightning path on the time delay evaluation was demonstrated. Previous modeling of a lightning path was carried out by Wan et al. [3] and Pulgarín et al. [1]. Wan et al. propose a comprehensive lightning path model with an accurate geometry. Pulgarín et al. model a lightning channel based on electromagnetic concept with a tortuosity properties. In the current work, a complicated path, such as that shown in Figures 14 and 17, was used. The effect of lightning path on the captured delay times is similar to what is reported by Pulgarin.

The locus of error in the short baseline TOA model is mapped in Figure 21. Unfortunately, there are no previous similarly reported results. The results presented in the current work complement the research work carried out by Mardiana and Kawasaki [30] for their proposed lightning locating system using the short baseline technique with three broadband antennas. In the current work, the azimuth and elevation angle equations ((20) and (21)) used by Tantisattayakul et al. or Mardiana and Kawasaki were shown to be influenced by many lightning parameters. The evaluation demonstrated in Figures 11 and 12 shows that $\alpha$ has a large error when the lightning leader is at a high elevation. In contrast, the maximum error of $\beta$ occurs when the leader is at a low elevation. In other words, the proposed TOA model fails under both conditions.

In short, the current work has successfully shown the development of a new and complete lightning simulation model taking into consideration many factors such as the effects of various lightning leader parameters. It also 
addresses the transmission of signals from the lightning leader and the capturing of those signals by a faraway detecting sensor in the form of two short baselines. It also analyses the pattern of arrival and the accuracy of the computed azimuth and elevation angles.

\section{Conclusion}

Simulations of lightning signal and its subsequent detection by sensors for the purpose of modelling the time of arrival (TOA) method of lightning locating system using three broadband antennas had been done. Consequently, based on the developed model, the influences of geometric parameters such as lightning path shape, distance of the leader, and leader location on the accuracy of the model are evaluated. The evaluation shows that the parameter, leader distance, has a direct effect on the elevation calculation accuracy in the order of hundreds of millidegrees, which can be defined as a significant value of hundreds of meters of leader distance. Furthermore, the results show that the variation in the leader shape path is a key factor in the procedure of finding the delay time especially in a method such as cross-correlation. Moreover, it is shown that the TOA technique except in the first quadrant fails in some locus of the three other quadrants because of the mathematical limitation of the model. In this study, three broadband antennas with a short baseline configuration were used for lightning detection. The configuration only gives the information of the azimuth and elevation. To mathematically obtain the location or coordinate of a lightning source, for example, a return stroke, a fourth antenna is necessary. Reconstructing the simulation to evaluate the new 4 -antenna configuration is an opportunity for future research. In addition, the use of a multistation system, with at least two separate short baseline stations in front of each other, or even a station for each quadrant, can be further explored.

\section{Conflict of Interests}

The authors declare that there is no conflict of interests regarding the publication of this paper.

\section{Acknowledgments}

The authors wish to thank the Ministry of Science, Technology and Innovation (MOSTI), Ministry of Education (MOE), and Universiti Teknologi Malaysia (Research Vote nos. 4S045, 03H59, and 4F291) for the financial aid.

\section{References}

[1] J. Pulgarín, C. Younes, and D. del Río, "Electromagnetic fields generated by lightning channels with tortuosity and its effect on the time-of-arrival technique," in Proceedings of the 12th International Symposium on Lightning Protection (SIPDA '13), pp. 140-144, October 2013.

[2] D. W. Clifford, K. E. Crouch, and E. H. Schulte, "Lightning simulation and testing," IEEE Transactions on Electromagnetic Compatibility, vol. 24, no. 2, pp. 209-224, 1982.
[3] H.-J. Wan, G.-H. Wei, and Q. Chen, "Three-dimensional numerical simulation of lightning discharge based on DBM model," in Proceedings of the 3rd IEEE International Conference on Computer Science and Information Technology (ICCSIT '10), pp. 520-523, 2010.

[4] F. Delfino, R. Procopio, M. Rossi, F. Rachidi, and C. A. Nucci, "An algorithm for the exact evaluation of the underground lightning electromagnetic fields," IEEE Transactions on Electromagnetic Compatibility, vol. 49, no. 2, pp. 401-411, 2007.

[5] Y. Baba and V. A. Rakov, "Applications of the FDTD method to lightning electromagnetic pulse and surge simulations," in Proceedings of the International Conference on Lightning Protection (ICLP '14), pp. 325-339, IEEE, Shanghai, China, October 2014.

[6] X. Liu, X. Cui, and L. Qi, "Calculation of lightning-induced overvoltages on overhead lines based on DEPACT macromodel using circuit simulation software," IEEE Transactions on Electromagnetic Compatibility, vol. 54, no. 4, pp. 837-849, 2012.

[7] K. Aniserowicz and T. Maksimowicz, "Comparison of lightning-induced current simulations in the time and frequency domains using different computer codes," IEEE Transactions on Electromagnetic Compatibility, vol. 53, no. 2, pp. 446-461, 2011.

[8] D. Prost, F. Issac, T. Volpert, W. Quenum, and J.-P. Parmantier, "Lightning-induced current simulation using RL equivalent circuit: application to an aircraft subsystem design," IEEE Transactions on Electromagnetic Compatibility, vol. 55, no. 2, pp. 378-384, 2013.

[9] Z. Song and M. R. Raghuveer, "Numerical simulation of lightning-caused inrush currents in power distribution transformers," in Proceedings of the Annual Report Conference on Electrical Insulation and Dielectric Phenomena (CEIDP '05), pp. 289-292, IEEE, October 2005.

[10] W. R. Gamerota, J. O. Elismé, M. A. Uman, and V. A. Rakov, "Current waveforms for lightning simulation," IEEE Transactions on Electromagnetic Compatibility, vol. 54, no. 4, pp. 880888, 2012.

[11] X.-M. Shao and A. R. Jacobson, "Model simulation of very lowfrequency and low-frequency lightning signal propagation over intermediate ranges," IEEE Transactions on Electromagnetic Compatibility, vol. 51, no. 3, pp. 519-525, 2009.

[12] S. Kato, T. Narita, T. Yamada, and E. Zaima, "Simulation of electromagnetic field in lightning to tall tower," in Proceedings of the 11th International Symposium on High Voltage Engineering, pp. 59-62, IET, August 1999.

[13] G. G. Gutierrez, E. P. Gil, D. G. Gomez, and J. P. Gomez, "Finitedifference time-domain method applied to lightning simulation and aircraft certification process," in Proceedings of the EMC Europe York, pp. 750-755, IEEE, September 2011.

[14] C. Yao, H. Wu, Y. Mi, Y. Ma, Y. Shen, and L. Wang, "Finite difference time domain simulation of lightning transient electromagnetic fields on transmission lines," IEEE Transactions on Dielectrics and Electrical Insulation, vol. 20, no. 4, pp. 1239-1246, 2013.

[15] A. J. Phillips and J. G. Anderson, "Computer simulation of lightning flashes to transmission lines," in Proceedings of the 11th International Symposium on High Voltage Engineering, Conference Publication no. 467, pp. 224-227, IET, 1999.

[16] Z. Jiang, F. Lin, L. Dai et al., "Simulation model of lightning stroke to a transmission line considering the leader propagation randomicity," in Proceedings of the 8th International Power Engineering Conference (IPEC '07), pp. 1282-1286, December 2007. 
[17] S. V. Mashak, H. N. Afrouzi, and Z. Abdul-Malek, "Simulation of lightning flash in time of arrival (toa) method by using three broadband antennas," in Proceedings of the 5th UKSim European Symposium on Computer Modeling and Simulation (EMS '11), pp. 287-292, IEEE, Madrid, Spain, November 2011.

[18] S. V. Mashak, H. N. Afrouzi, and Z. Abdul-Malek, "Simulation of lightning flash and detection using time of arrival method based on four broadband antennas," in Proceedings of the 1st International Conference on Informatics and Computational Intelligence (ICI '11), pp. 301-305, December 2011.

[19] B. Salimi, Z. Abdul-Malek, S. J. Mirazimi, and K. Mehranzamir, "Investigation of short base line lightning detection system by using time of arrival method," in Intelligent Informatics, vol. 182 of Advances in Intelligent Systems and Computing, pp. 141-147, Springer, Berlin, Germany, 2013.

[20] B. Salimi, Z. Abdul-Malek, K. Mehranzamir, S. V. Mashak, and H. N. Afrouzi, "Localised single-station lightning detection by using TOA method," Jurnal Teknologi, vol. 64, no. 4, pp. 73-77, 2013.

[21] R. Roberts, “Tdoa localization techniques," Working Group for Wireless Personal Area Networks (WPANs), 2004.

[22] R. L. Dowden, J. B. Brundell, and C. J. Rodger, "VLF lightning location by time of group arrival (toga) at multiple sites," Journal of Atmospheric and Solar-Terrestrial Physics, vol. 64, no. 7, pp. 817-830, 2002.

[23] V. Mazur, E. Williams, R. Boldi, L. Maier, and D. E. Proctor, "Initial comparison of lightning mapping with operational timeof-arrival and interferometric systems," Journal of Geophysical Research D: Atmospheres, vol. 102, no. 10, pp. 11071-11085, 1997.

[24] W. I. Ibrahim and Z. A. Malek, "Time-to-thunder method of lightning distance determination," in Proceedings of the IEEE International Conference on Power and Energy (PECon '10), pp. 357-362, IEEE, Kuala Lumpur, Malaysia, December 2010.

[25] Z. Abdul-Malek, Z. Adzis, A. Novizon, and N. Abdullah, "Waveform and location analyses of localised lightning locating system," in Proceedings of the International Conference on High Voltage Engineering and Application (ICHVE '10), pp. 132-135, New Orleans, La, USA, October 2010.

[26] W. I. Ibrahim, M. R. Ghazali, S. A. Ghani, and Z. A. Malek, "Measurement of vertical electric fields from lightning flashes using parallel plate antenna," in Proceedings of the 1st International Conference on Electrical, Control and Computer Engineering (InECCE '11), pp. 466-471, June 2011.

[27] P. Kułakowski, J. Vales-Alonso, E. Egea-López, W. Ludwin, and J. García-Haro, "Angle-of-arrival localization based on antenna arrays for wireless sensor networks," Computers and Electrical Engineering, vol. 36, no. 6, pp. 1181-1186, 2010.

[28] T. Tantisattayakul, K. Masugata, I. Kitamura, and K. Kontani, "Broadband VHF sources locating system using arrival-time differences for mapping of lightning discharge process," Journal of Atmospheric and Solar-Terrestrial Physics, vol. 67, no. 11, pp. 1031-1039, 2005.

[29] D. Cao, X. Qie, S. Duan, J. Yang, and Y. Xuan, "Observations of VHF source radiated by lightning using short baseline technology," in Proceedings of the Asia-Pacific Symposium on Electromagnetic Compatibility (APEMC '10), pp. 1162-1165, April 2010.

[30] R. Mardiana and Z. Kawasaki, "Broadband radio interferometer utilizing a sequential triggering technique for locating fastmoving electromagnetic sources emitted from lightning," IEEE Transactions on Instrumentation and Measurement, vol. 49, no. 2, pp. 376-381, 2000.
[31] R. W. Klebesadel, W. Doyle Evans, E. E. Fenimore, J. G. Laros, and J. Terrell, "Time-of-arrival location technique," Los Alamos Science, vol. 3, pp. 10-23, 1982.

[32] V. A. Rafalsky, A. P. Nickolaenko, A. V. Shvets, and M. Hayakawa, "Location of lightning discharges from a single station," Journal of Geophysical Research: Atmospheres, vol. 100, no. 10, pp. 20829-20838, 1995.

[33] P. Wagenaars, P. A. A. F. Wouters, P. C. J. M. van der Wielen, and E. F. Steennis, "Accurate estimation of the time-of-arrival of partial discharge pulses in cable systems in service," IEEE Transactions on Dielectrics and Electrical Insulation, vol. 15, no. 4, pp. 1190-1199, 2008.

[34] M. R. Spiegel, Mathematical Handbook of Formulas and Tables, 1968.

[35] N. A. Ahmad, M. Fernando, Z. A. Baharudin, V. Cooray, H. Ahmad, and Z. Abdul Malek, "Characteristics of narrow bipolar pulses observed in Malaysia," Journal of Atmospheric and SolarTerrestrial Physics, vol. 72, no. 5-6, pp. 534-540, 2010. 

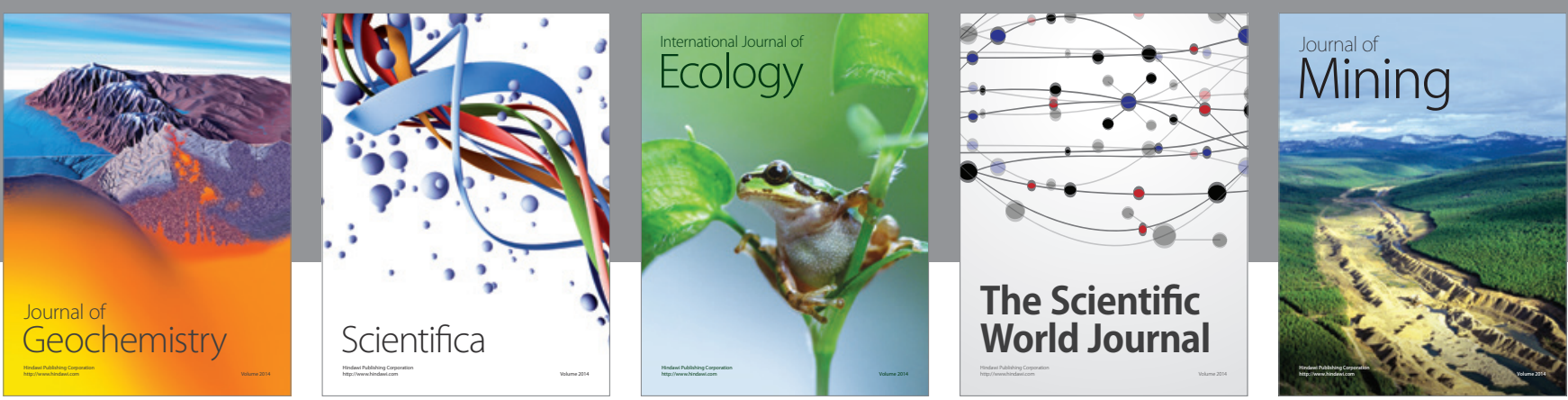

The Scientific World Journal
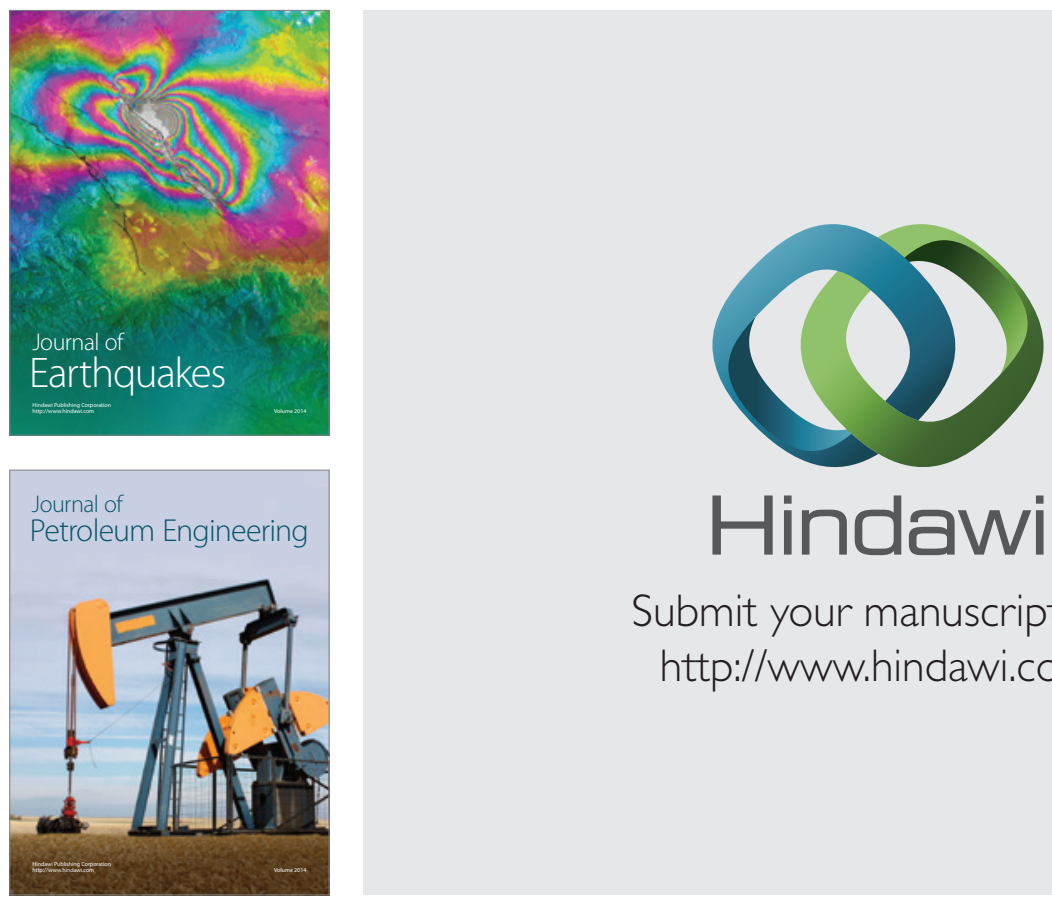

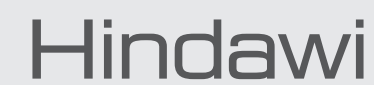

Submit your manuscripts at

http://www.hindawi.com
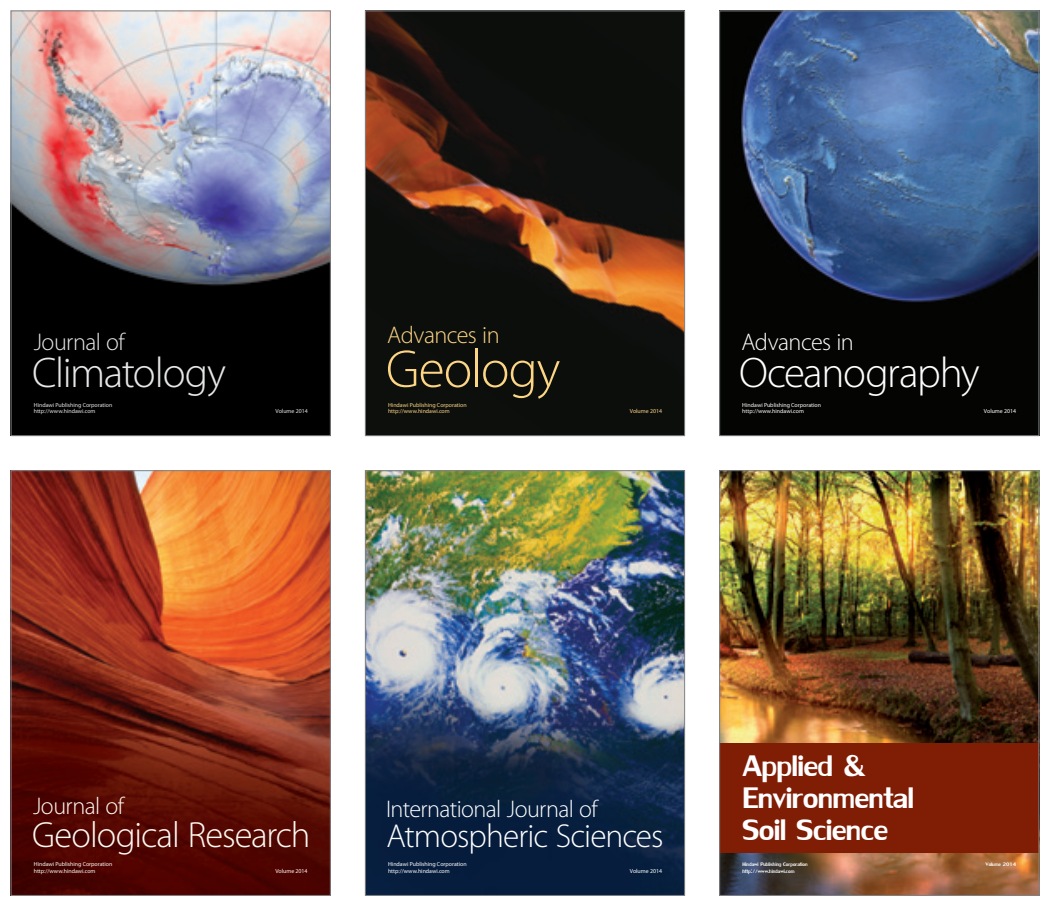
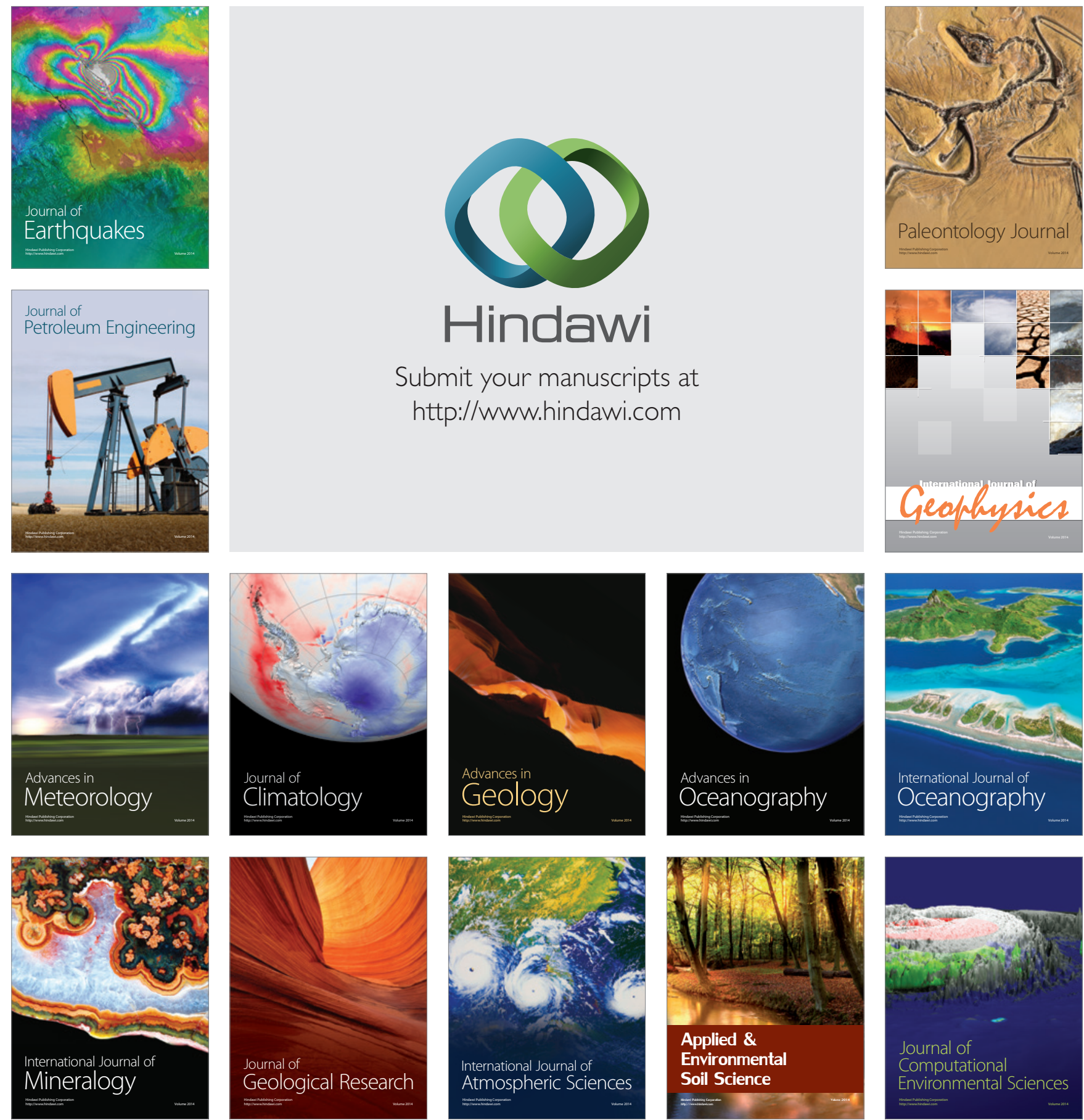